\title{
Ferrodistortive Instability at the (001) Surface of Half-Metallic Manganites
}

\author{
J. M. Pruneda, ${ }^{1,2}$ V. Ferrari, ${ }^{3,4}$ R. Rurali, ${ }^{5}$ P. B. Littlewood,${ }^{3}$ N. A. Spaldin, ${ }^{6}$ and Emilio Artacho ${ }^{7}$ \\ ${ }^{1}$ Department of Physics, University of California, Berkeley, California 94720, USA \\ ${ }^{2}$ Institut de Ciència de Materials de Barcelona, (ICMAB-CSIC), Campus de la UAB, 08193 Bellaterra, Spain \\ ${ }^{3}$ Cavendish Laboratory, University of Cambridge, Madingley Road, Cambridge CB3 OHE, United Kingdom \\ ${ }^{4}$ Departamento de Física, Comisión Nacional de Energía Atómica, Gral. Paz 1499, 1650 San Martín, Buenos Aires, Argentina \\ ${ }^{5}$ Departament d'Enginyeria Electrònica, Universitat Autònoma de Barcelona, 08193 Bellaterra, Spain \\ ${ }^{6}$ Materials Department, University of California, Santa Barbara, California 93106-5050, USA \\ ${ }^{7}$ Department of Earth Sciences, University of Cambridge, Downing Street, Cambridge CB2 3EQ, United Kingdom
}

(Received 2 May 2007; published 26 November 2007)

\begin{abstract}
We present the structure of the fully relaxed (001) surface of the half-metallic manganite $\mathrm{La}_{0.7} \mathrm{Sr}_{0.3} \mathrm{MnO}_{3}$, calculated using density functional theory. Two relevant ferroelastic order parameters are identified and characterized. The known tilting of the oxygen octahedra, which is present in the bulk phase, decreases towards the surface. A ferrodistortive Mn off-centering, triggered by the surface and not reported before, decays monotonically into the bulk. This distortion affects neither the half-metallicity nor the zero-temperature magnetization, but does change the effective spin-spin interactions, and thus the temperature dependence of the magnetic properties.
\end{abstract}

DOI: $10.1103 /$ PhysRevLett.99.226101

PACS numbers: 75.70. $-\mathrm{i}, 73.20 .-\mathrm{r}$, 75.47.Lx

A delicate balance between structure and physical properties in perovskites makes this family of materials interesting scientifically and of technological importance. In addition to high- $T_{C}$ superconductors, most of the inorganic ferroelectric and piezoelectric materials belong to this family, with an important presence in the growing field of multiferroics [1]. Manganites are prominent members of this family since the discovery of colossal magnetoresistance [2]. Within an extremely rich phase diagram, the optimally doped $\mathrm{La}_{1-x} A_{x} \mathrm{MnO}_{3}(A=\mathrm{Sr}$, Ca) phase, obtained for $x \sim 0.3$, is of particular interest because of its half-metallicity, i.e., with a gap for one spin direction and thus with fully spin-polarized carriers, extremely useful for spintronics.

A fundamental obstacle for the development of manganite devices is the fact that some of the physical properties of the surface markedly deviate from those of the bulk. Electron-lattice interactions are known to play an important role in the electronic properties of these oxides [3], making it thus crucial to characterize the structural distortions at the surface, which are so far unknown. The primary goal of this work is to determine the structural changes induced by surface termination at the (100) surface of $\mathrm{La}_{0.7} \mathrm{Sr}_{0.3} \mathrm{MnO}_{3}$ (LSMO) and the influence of these structural changes on the electronic properties for the clean surface. The effect of oxygen vacancies, as prototypical imperfections, is briefly discussed at the end.

The theoretical description of manganites is challenging given the strong correlations of the transition metal $d$ electrons, as well as the complex chemistry and large range of possible structural distortions characteristic of perovskites. A $b$ initio studies to date have been confined to experimental crystal parameters or pseudocubic structures $[4,5]$, partly because a full description of structure and dop- ing represents a substantial increase of the computational needs, but also because the lattice parameters obtained at the time were in poor agreement with experiments.

Here, we present $a b$ initio results for a fully relaxed LSMO (001) surface obtained using density-functional theory (DFT) within the generalized gradient approximation (GGA). Our choice of exchange-correlation functional was motivated by our recent GGA calculations for bulk LSMO [6], in which we found good agreement between the theoretically optimized pseudocubic and experimental lattice constants, and qualitatively correct half-metallic electronic behavior. Since our focus is on structural, rather than on electronic properties, and since we are interested in the metallic phase of LSMO, which reduces the errors related to the nonlocality of the exchange-correlation hole, we elect not to include higher order descriptions of correlation effects such as in DFT $+U$ [5]. Indeed, it was recently shown [7] that the use of GGA $+U$ does not significantly change the structural properties. However, the limitations of today's GGAs for treating highly-correlated systems should be kept in mind, in particular, when we discuss changes in the surface electronic spectral properties based on Kohn-Sham eigenvalues [8].

Our calculations were performed using the SIESTA implementation of DFT [9,10]. The spin-polarized gradientbased PBE exchange-correlation functional was used [11], with a grid cutoff of 320 Ry for integrations in real space. Semicore states were explicitly included in the calculations. Details of the pseudopotentials and numerical atomic orbitals are given in Ref. [6]. Atomic positions were relaxed until the forces were smaller than $25 \mathrm{meV} / \AA$.

To validate our method in obtaining relaxed atomic structures, we first studied the properties of bulk LSMO. We built a cell that is a $\sqrt{2} \times \sqrt{2} \times 2$ repetition of the ideal 
perovskite cubic cell, and performed a variable cell relaxation of the crystal structure. Our calculated lattice parameters are within $2 \%$ of the experimentally reported values, and our Mn-O bond lengths $(1.96 \AA)$ are even closer to experiment $(1.95 \AA)$ [12]. The tilt angle $\left(6.6^{\circ}\right)$ that we obtain for the optimized structure is in excellent agreement with the experimental value $\left(6.8^{\circ}[12]\right)$. Since these distortions are believed to be the source of the strong electronphonon coupling in manganites [3] (they lift the degeneracy of $e_{g}$ levels, as recently shown in photoemission experiments [13], and reduce the electronic energy for single occupancy of this state) their accurate representation in our calculations is essential.

The full description of distortions and doping requires the use of a large supercell: 224 atoms, in $4 \times 2$ repetitions of the original cubic cell in the surface $a b$-plane, eleven atomic layers (5.5 unit cells) along the direction perpendicular to the surface, and $\mathrm{MnO}_{2}$ termination at both sides of the slab [14]. This thickness ( $\gtrsim 2 \mathrm{~nm}$ ) is comparable to the ones used in experimental studies of strained manganites [15]. The electronic structure at the center of the slab is practically indistinguishable from the bulk.

We begin with a pseudocubic structure and allow for full atomic relaxations, while keeping the in-plane lattice parameters fixed to the relaxed bulk values. The supercell is large enough to accommodate the octahedral tiltings, and commensurate with the orthorhombic phase in the $a b$-plane. The surface breaks the bulk rhombohedral symmetry, most importantly the threefold axes of the $R \overline{3} c$ phase, therefore falling back onto the Pnma tilt system. Doping is then introduced explicitly by substituting $\mathrm{La}$ atoms by $\mathrm{Sr}$, with the resulting structure having a quasirandom distribution of $13 \mathrm{Sr}$ atoms and $27 \mathrm{La}$ atoms.

We find that relaxation of the atomic positions gives rise to a buckling in the surface layer, with the Mn atoms shifted out of the plane. There is also a change in the octahedral tiltings and of the $\mathrm{Mn}-\mathrm{O}$ bonding distances. In Fig. 1(a), we plot the tilting angle of the $\mathrm{MnO}_{6}$ octahedra as a function of the position of the Mn layer across the slab. It can be seen that the tilting at the surface is considerably reduced with respect to the bulk value (dashed line). In addition, we find that the buckling of the $\mathrm{Mn}$ atoms with respect to oxygens in the surface layer propagates to the subsurface giving rise to an off-centering similar to that of ferroelectric perovskites. The magnitude of this offcentering, shown in Fig. 1(b), decays monotonically towards the center of the slab, where it is zero due to the cancelling effect of both surfaces. The distortion results in important changes in the Mn-O bonding distances along the $z$ direction, whereas the in-plane bonds remain essentially unchanged.

Although the Mn-O buckling at the surface layer is a result of local surface chemistry (see the surface-state shift below), the off-centering deeper into the bulk is not. The situation is better described as a polar, ferroelastic insta-

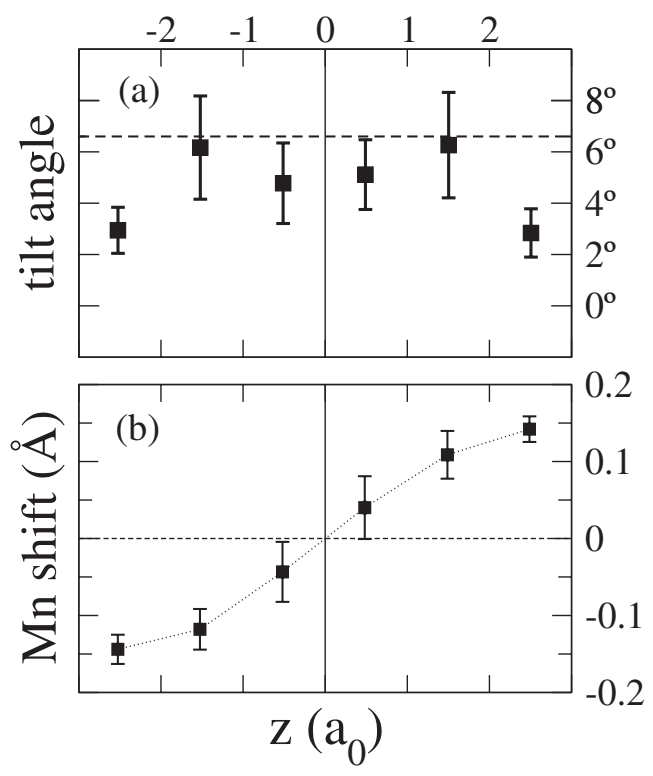

FIG. 1. (a) Octahedral tilt angle versus position across the slab, $z$, in units of the cubic lattice constant, $a_{0}(=3.89 \AA)$. The dashed line shows the value for the theoretically relaxed bulk geometry. (b) Off-centering (in $\AA$ ) of the $\mathrm{Mn}$ atoms from the plane defined by the neighboring $\mathrm{O}$ atoms in the $\mathrm{MnO}_{2}$ layer. The $z$ origin is defined at the center of the slab. Error bars indicate the differences within the same atomic layer.

bility, which is triggered by the surface buckling. Since this instability is not present in the bulk phase, it therefore decays from the surface into the bulk. Such surfacetriggered decaying ferroelastic distortions are described in the literature [16], and can be very slowly decaying [17]. Our calculations, however, allow us to ascertain only that the decay length $\lambda_{o}$ is not smaller than $\sim 10 \AA$, since the antisymmetric character of the order parameter [Fig. 1(b)] forces it to go through zero at the center of the slab.

The length-scale of the decay towards the surface of the octahedral tilt order parameter, $\lambda_{t}$, should be captured in our calculations since the symmetry of the slab does not constrain it in this case. We find that it is of the order of a few atomic layers. However, we can not rule out the possibility that both order parameters are coupled at the surface, whereby $\lambda_{t}$ would be in fact determined by the constraint on $\lambda_{o}$. Indeed, couplings and/or competitions between polar ferroelectric modes and tiltings and rotations of oxygen octahedra are well known to occur in related perovskites [18-20]. The decay of the untilting seems oscillatory and commensurate with the crystal, but not much can be concluded here since this behavior is barely resolved within the error bars. Both monotonic and oscillatory behaviors can be expected in surface ferroelastic decays [16].

In order to understand the surface buckling and assess its effect on the half-metallicity, we briefly compare the bulk 
and surface electronic structures within the GGA. Figure 2 shows our calculated Mn $3 d$ partial densities of states (DOS) in the center of the slab (a), and for unrelaxed (b) and relaxed (c) surface configurations. At the center of the slab, the system is bulklike and half-metallic, with $e_{g}$ character for the states at $\varepsilon_{F}$, and localized $t_{2 g}^{\dagger}$ states around $1.5 \mathrm{eV}$ below, in fair agreement with previous calculations [4] and photoemission experiments [21]. Importantly, this half-metallicity persists at the surface, and since the majority spin metallicity results from a broad Mn $3 d-\mathrm{O} 2 p$ hybridized band (the $\mathrm{Mn} d_{z^{2}}$ and $d_{x^{2}-y^{2}}$ states, as well as the $p_{z}$ of the oxygen underneath the Mn, dominate the Fermi level at the surface) it is likely to be accurately represented in our GGA methodology. For the unrelaxed (cubic) structure, the surface termination induces a localized surface state, of mainly $d_{z^{2}}$ and $d_{x y}$ character. This state had been previously observed in localspin-density and self-interaction-corrected calculations
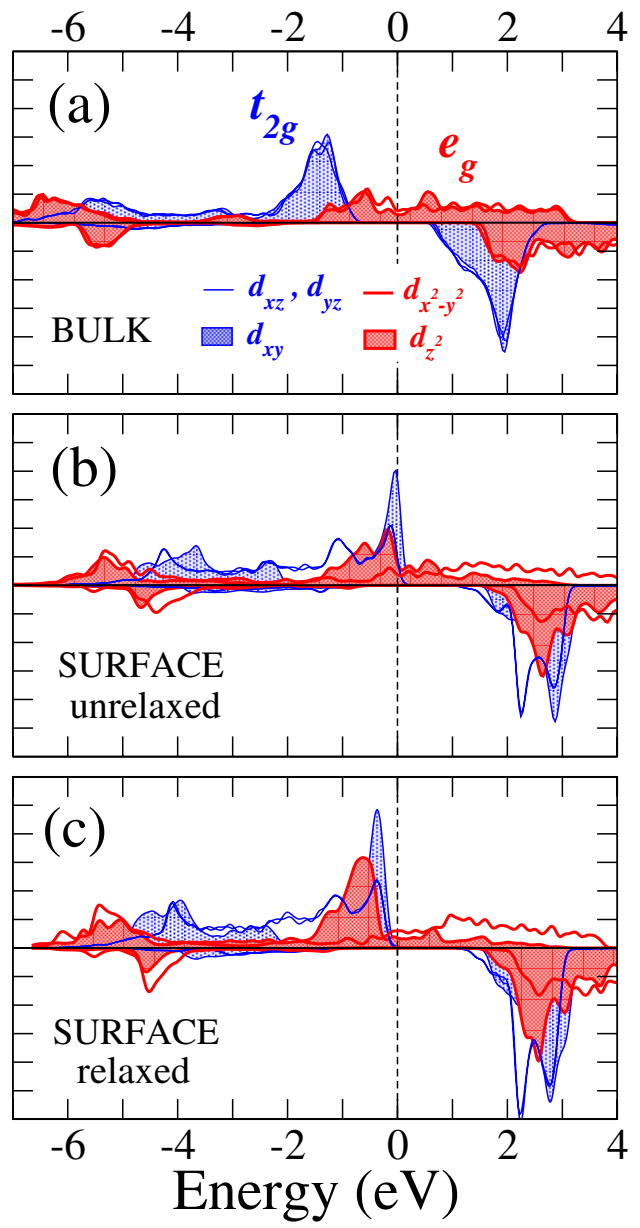

FIG. 2 (color online). Partial density of states for up (down) spins [bottom (top)] for the Mn $3 d$ orbitals in the center of the slab (a), and the surface layer before (b), and after (c) the structural relaxation. The surface state is mainly composed of $d_{z^{2}}$ and $d_{x y}$ orbitals, which are shown as shaded regions. with unrelaxed cubic surface geometries, and originates after the splitting of the states $e_{g} \rightarrow d_{z^{2}}, d_{x^{2}-y^{2}}$, and $t_{2 g} \rightarrow$ $d_{x y}\left(d_{y z}, d_{x z}\right)$, related to the surface-induced symmetry breaking, from cubic $O_{h}$ to $C_{4 v}$ [22,23]. The surface state shifts to lower energies away from $\varepsilon_{F}$ when the geometry relaxes. This is the driving force for the surface buckling.

Concerning magnetic properties, we observe that the zero $\mathrm{K}$ magnetic moment remains essentially unaltered up to the very surface, with a value of $3.66 \mu_{B}$ per $\mathrm{Mn}$ atom at the relaxed surface compared with $3.53 \mu_{B}$ in the bulk. The ground state remains ferromagnetic. In order to address the finite temperature magnetism, we explored different magnetic configurations and found that the energetic cost of reversing one magnetic moment at the surface is reduced to $30 \%$ of the bulk value, indicative of lower effective magnetic interactions, and in line with experimental results [24]. Forces on the atoms of up to $1.54 \mathrm{eV} / \AA(1.01 \mathrm{eV} / \AA)$ have been found upon single spin reversal in the surface (in the bulk), indicating (i) that the surface ferromagnetic coupling would weaken even more as compared to the bulk, and (ii) a possible important magnon-phonon coupling.

Two competing considerations arise when relating the magnetic results to the structural ones. The doubleexchange (DEX) mechanism, in which localized $3 d t_{2 g}$ electron spins interact ferromagnetically through the $e_{g}$ conduction-electron hopping between nearest neighbors, is frequently used to describe the electronic properties of manganites. On one hand, the electron-hopping energy among $\mathrm{Mn} e_{g}$ states in this DEX model is expected to decrease with the bending of the Mn-O-Mn bonds, in agreement with the observed reduction of the ferromagnetic Curie temperature $T_{M}$ in doped manganites [25]. The octahedral untilting towards the surface discussed before would thus suggest a surface enhancement of hopping and $T_{M}$, at odds with the mentioned observations. On the other hand, however, the hopping energy should be much more affected by the substantial off-centering, thus altering the interlayer coupling.

The surface-triggered ferrodistortive instability discussed above is very similar in kind and magnitude to that of ferroelectrics like $\mathrm{BaTiO}_{3}$. It is thus tempting to consider multiferroic possibilities for this system. Considering the calculated values for the atomic displacements, and taking $\sim+3 e$ for the nominal charge of $\mathrm{Mn}$, we would obtain a polarization of $\sim 10 \mu \mathrm{C} / \mathrm{cm}^{2}$ at the surface layer, which would be larger than the values obtained for other orthorhombic perovskite manganite multiferroics [26]. It must be remembered, however, that it is a metallic system, and thus any polarization will be screened by the itinerant carriers. In addition to the ferroelastic characteristics discussed above, the surface buckling could display surface piezoelectric signatures, considering the Thomas-Fermi screening length of this (poor) metal, affecting the work function and its strain dependence. This 
could be a relevant consideration for understanding the orientation of dipolar molecules deposited on the surface. Spin valve devices based in LSMO have already been demonstrated with Alq3 (8-hydroxy-quinoline aluminum) [27], and a substantial shift in its molecular energy levels has been measured [28]. Our results suggest that the observed lowering of the molecular energy levels is caused by the electrostatic potential resulting from the orientation of the Alq3 molecular dipole by the LSMO surface dipole.

It should be remembered that these results are for the defect-free $\mathrm{MnO}_{2}$-terminated LSMO surface. They may be very different for $(\mathrm{La}, \mathrm{Sr}) \mathrm{O}$ termination [23] and, most importantly, in the presence of defects (dopant segregation or oxygen vacancies). Recent photoemission experiments have suggested the presence of strongly localized $\mathrm{Mn}^{+2}$ states at the surface of LSMO, related to oxygen vacancies $\left(V_{\mathrm{O}}\right)$ [29]. Additional DFT results for our LSMO slab reveal that the ferroelastic distortions are substantially altered around a $V_{\mathrm{O}}$. For a $V_{\mathrm{O}}$ between surface and subsurface $\mathrm{Mn}$ atoms, the surface buckling is enhanced by $0.1 \AA$, and the off-centering of the subsurface $\mathrm{Mn}$ is depressed by $0.1 \AA$. A resonance peak appears in the unoccupied minority-spin DOS, with most of its weight at the $d_{z 2}$ of the closest surface $\mathrm{Mn}$ atom. It remains, however, $0.9 \mathrm{eV}$ above the bottom of the minority-spin conduction band. Similarly, none of the vacancies studied destroyed the half metallicity of LSMO (in agreement with [29]). Full details will be discussed elsewhere [30].

In conclusion, our first-principles structural relaxation of the LSMO (001) surface has revealed a surprising surfacetriggered ferroelastic instability of an off-centering ferrodistortive kind. The distortion is driven by the surface electronic structure, and causes a weakening of the tendency to magnetic order close to the surface. We hope that our observations will stimulate further work towards the characterization of the discussed instabilities, as well as its possible exploitation and its coupling to the magnetic behavior.

We acknowledge discussions with E. K. H. Salje, L.E. Hueso, M. A. Carpenter, L. Brey, and N. D. Mathur. The calculations were done at the HPCF of Cambridge University. This work was funded by the UK EPSRC, CONICET-Argentina (V.F.), NERC, BNFL, the UE Marie Curie Fellowship (J.M.P.), and the National Science Foundation's Division of Materials Research, Grant No. DMR-0605852 (N. A. S.). N. A. S. thanks the Miller Institute at UC Berkeley for their support during this work.

[1] W. Eerenstein, N. Mathur, and J. Scott, Nature (London) 442, 759 (2006).

[2] K. Dörr, J. Phys. D: Appl. Phys. 39, R125 (2006).

[3] A. J. Millis, Nature (London) 392, 147 (1998).
[4] W.E. Pickett and D. J. Singh, Phys. Rev. B 53, 1146 (1996).

[5] S. Satpathy, Z. S. Popovic, and F. R. Vukajlovic, Phys. Rev. Lett. 76, 960 (1996).

[6] V. Ferrari, J. M. Pruneda, and E. Artacho, Phys. Status Solidi A 203, 1437 (2006).

[7] C. Ma, Z. Yang, and S. Picozzi, J. Phys. Condens. Matter 18, 7717 (2006).

[8] G. Kotliar, S. Y. Savrasov, K. Haule, V. S. Oudovenko, O. Parcollet, and C. A. Marianetti, Rev. Mod. Phys. 78, 865 (2006).

[9] P. Ordejón, E. Artacho, and J. M. Soler, Phys. Rev. B 53, R10441 (1996).

[10] J. M. Soler, E. Artacho, J. D. Gale, A. García, J. Junquera, P. Ordejón, and D. Sánchez-Portal, J. Phys. Condens. Matter 14, 2745 (2002).

[11] J. P. Perdew, K. Burke, and M. Ernzerhof, Phys. Rev. Lett. 77, 3865 (1996).

[12] P. G. Radaelli, G. Iannone, M. Marezio, H. Y. Hwang, S.-W. Cheong, J.D. Jorgensen, and D. N. Argyriou, Phys. Rev. B 56, 8265 (1997).

[13] M. C. Falub, M. Shi, P. R. Willmott, J. Krempasky, S. G. Chiuzbaian, K. Hricovini, and L. Patthey, Phys. Rev. B 72, 054444 (2005).

[14] M. Yoshimoto, H. Maruta, T. Ohnishi, K. Sasaki, and H. Koinuma, Appl. Phys. Lett. 73, 187 (1998).

[15] M. Ziese, H.C. Semmelhack, K. H. Han, S. P. Sena, and H. J. Blythe, J. Appl. Phys. 91, 9930 (2002).

[16] B. Houchmandzadeh, J. Lajzerowicz, and E. Salje, J. Phys. Condens. Matter 4, 9779 (1992).

[17] S. A. Hayward, F. D. Morrison, S. A. T. Redfern, E. K. H. Salje, J. F. Scott, K. S. Knight, S. Tarantino, A. M. Glazer, V. Shuvaeva, and P. Daniel et al., Phys. Rev. B 72, 054110 (2005).

[18] P. M. Woodward, Acta Crystallogr. Sect. B 53, 32 (1997).

[19] P. M. Woodward, Acta Crystallogr. Sect. B 53, 44 (1997).

[20] D. I. Bilc and D. J. Singh, Phys. Rev. Lett. 96, 147602 (2006).

[21] A. Chikamatsu, H. Wadati, H. Kumigashira, M. Oshima, A. Fujimori, N. Hamada, T. Ohnishi, M. Lippmaa, K. Ono, and M. Kawasaki et al., Phys. Rev. B 73, 195105 (2006).

[22] A. Filippetti and W.E. Pickett, Phys. Rev. B 62, 11571 (2000).

[23] H. Zenia, G.A. Gehring, G. Banach, and W. M. Temmerman, Phys. Rev. B 71, 024416 (2005).

[24] J.-H. Park, E. Vescovo, H.-J. Kim, C. Kwon, R. Ramesh, and T. Venkatesan, Nature (London) 392, 794 (1998).

[25] J. Fontcuberta, B. Martínez, A. Seffar, S. Piñol, J. L. García-Muñoz, and X. Obradors, Phys. Rev. Lett. 76, 1122 (1996).

[26] T. Kimura, T. Goto, H. Shintani, K. Ishizaka, T. Arima, and Y. Tokura, Nature (London) 426, 55 (2003).

[27] Z. H. Xiong, D. Wu, Z. V. Vardeny, and J. Shi, Nature (London) 427, 821 (2004).

[28] Y.Q. Zhan, I. Bergenti, L.E. Hueso, V. Dediu, M.P. de Jong, and Z.S. Li, Phys. Rev. B 76, 045406 (2007).

[29] M.P. de Jong, I. Bergenti, W. Osikowicz, R. Friedlein, V. A. Dediu, C. Taliani, and W. R. Salaneck, Phys. Rev. B 73, 052403 (2006).

[30] R. Rurali et al. (to be published). 